\title{
Modeling of deformation phenomena in volume label during its operation
}

\begin{abstract}
BIt was considered in the article the research of physical and mechanical properties of materials based on PVC film ORACAL and RITRAMA that used in the production of volume labels. In particular, it was shown the changes of dependences between linear deformation of printed and unprinted films and the value of stress with regard to their minimum and maximum values. It was also established that deformation properties of the films are different in the transverse and longitudinal directions, which should be considered when producing labels. It was conducted the modeling of stress-strain state of volume label when gluing it to the surfaces of various shapes. We considered the boundary conditions for displacement and stress of labels layer components with regard to the chemical structure of epoxy resins and their physical and mechanical characteristics.
\end{abstract}

\section{KEY WORDS}

volume label-sticker, deformation, stress, modeling

\author{
Svitlana Sheludko', \\ Olga Ogirko ${ }^{2}$ \\ ${ }^{1}$ Ukrainian Academy of Printing, \\ Faculty of Publishing, Printing and \\ Information Technologies, \\ Lviv, Ukraine \\ 2Lviv State University of Internal \\ Affairs, Lviv, Ukraine
}

Corresponding author: Svitlana Sheludko e-mail:pmtp_uad@ukr.net

First recieved: 14.02.2017. Accepted: 14.03.2017.

\section{Introduction}

Today label is an integral part of the packaging of any product, which is serves as its identifier. Therefore, the goal of products manufacturers is not only improve the design of labels-stickers, but also the development of new technologies of their finishing and protection to enhance operational properties. Volume labels take a significant place in this sense. Polyurethane or epoxy resins of three types are usually used for the manufacture of volume labels in advertising and souvenir industry: a soft resin (for sticking to object of circular, cylindrical or irregular surfaces), solid resin (for labels, which are planned to stick on a hard surface), and medium hardness resin. It was formed a so-called "lens" with thickness 1.5-2.5 mm, which becomes transparent and flexible after polymerization through polymer composition (resins and the hardener in a certain proportion) deposited on top of the label printed image. Self-adhesive labels may have additional coverage, such as UV coating, lamination, foil stamping and more. Each method of such decoration was designed to provide special personality, more abrasion resistance and precision for product. The advantageous feature of these labels is their ability to self-regenerate, resistance to fading and mechanical damage, exposure to chemicals. Plastic polymer resin has the ability to recover its original shape, so that small scratches disappear after a few minutes, and it becomes smooth again (Rehbinder, 1978; Pocius, 2002; Sheludko, 2013).

The aim of research was to carry out a modeling of stress-strain state of volume labels produced on adhesive material- PVC film like "Oracal" and "RITRAMA" during its use and explore their physical and mechanical properties.

\section{Materials}

An important moment in volume labels manufacturing technology is the choice of resin. The choice of resin is determined by such parameters: initial components viscosity; viscosity of the obtained composition; mixing 
proportions; resin hardness after polymerization, and the necessary rigidity and polymer thickness, features of labels shape. Polymer coatings can have different stiffness, which determines the resistance of the coating to abrasion. It was established that the greater the viscosity of the resin, the greater the height of the lens can be formed on the surface of the label, which is important when applying polymer on large areas.

The choice of resin depends on the time of viability and full curing. Curing of resin become faster when higher its temperatures, it is easier to remove bubbles from it, but at the same time unfortunately decreases the viscosity and therefore the height of obtained lens will be smaller. The choice of self adhesive materials depends on the labels operating conditions and surface properties to which the label is applied. Resin polymerization occurs at room temperature for about 8 hours in a strictly horizontal position and in compliance with the humidity and temperature in the room. However, this time can be significantly reduced by using special infrared dryers.

It is important to prepare labels to fill. Due to the fact that all the filling machines designed to work with sheet material, as the object for filling was taken sheets at size $40 \times 40$ and $50 \times 70 \mathrm{~cm}$ with printed labels on it. In addition, the label must always be incised, such as cutting on plotters ROLAND GX-640. The presence of a clear boundary on the label needs for applied resin didn't flow of necessary image boundaries (Rehbinder, 1978; Pocius, 2002; Sheludko, 2013).

As objects of research it was selected volume labels digitally printed on large-format plotter Epson Sure Color SC-S70610 by using PVC film ORACAL 640 and RITRAMA filled with epoxy resin MTBJZJ MTB8000/7700 (hard resin) and MTB-3800/9213 (soft resin), it was used hardener Rich R-2265 (Figure 1).

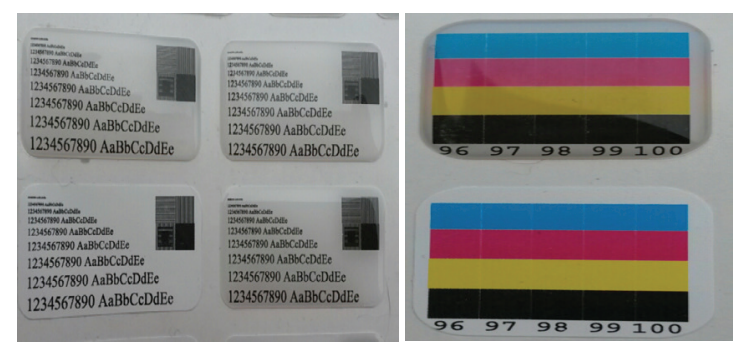

» Figure 1: Samples of volume labels filled with polymer based on soft (a) and hard (b) epoxy resin

Deformation properties of film materials ORACAL and RITRAMA determined on tensile machine 050/Rt-601U of Japanese firm "Kimura Mashinery" by GOST 14236-81 (GOST 14236-81, 1981). The research was carried out at the velocity of the clamp $100 \mathrm{~mm} / \mathrm{min}$ until breaking of the sample at $22^{\circ} \mathrm{C}$. According to scale available on the device it was fixed value of destroying force $(F, H)$ and by the known formulas determined the value of relative elongation of samples (in \%) and deformation (in $\mathrm{MPa}$ ).

ORACAL and RITRAMA are flexible calender monomeric PVC films. RITRAMA film has much larger internal residual stresses than ORACAL, through which it gives a significant shrinkage (more than $0.4 \mathrm{~mm}$ according to standard FINAT TM 14) during bonding on round surfaces. Film ORACAL has a silicon surface on the back. Mechanical properties of the films are important for their operation. Typical diagram of deformations for plastic materials contains four sections: elastic reverse deformation (1); beginning of irreversible deformation (2), when the material prevents the load; plastic deformation (3), when there is fluidity; formation of so-called "neck" of the samples (4), which corresponds to a small strengthening of the material and its destruction (Figure 2). During the formation of a neck in amorphous and crystalline polymers it is a transition from isotropic to anisotropic (oriented) state.

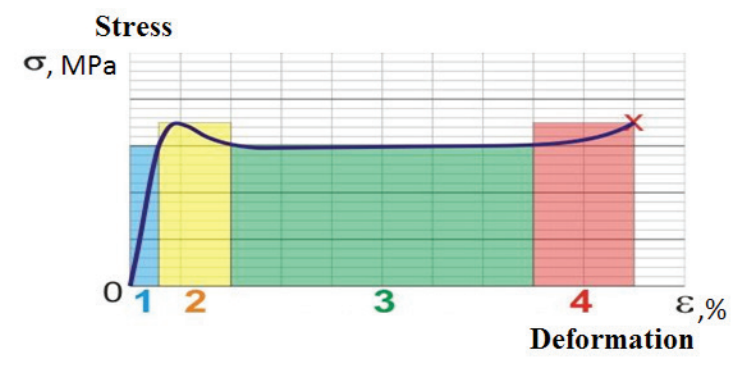

» Figure 2: Typical diagram of films deformations

This transition in crystalline polymers is accompanied by melting (destruction) of output crystalline regions under mechanical stress, orientation of macromolecules areas in the stretching direction and recrystallization. Stresses that correspond to the maximum on the curve of stretching crystalline polymer are called stresses of recrystallization.

\section{Results and discussion}

Based on the researches it was defined the dependence of linear deformation $\varepsilon$ and stress $\sigma$ for the investigated material. It was revealed that on the relative elongation of films and their breaking effort has considerable influence their direction (longitudinal or transverse), presence of the printed image (Table 1).

As can be seen, film RITRAMA-145 is able to withstand greater stresses and more resistant to fracture at 1.5 times than ORACAL. Breaking effort of film ORACAL (according to DIN EN ISO 527) is $19 \mathrm{MPa}$ (min) and 30.6 $\mathrm{MPa}(\max )$ in the longitudinal and $28.1 \mathrm{MPa}(\mathrm{min})$ and $46 \mathrm{MPa}$ ( $\mathrm{max}$ ) in transverse directions; elongation at break (according to DIN EN ISO 527) in the longitudinal direction is $\min 130 \%$, transverse direction- $\min 170 \%$ for film ORACAL. Elongation at break is $245 \%$ and $275 \%$ 


\section{Table 1}

Properties of the unprinted cardboard $\left(\mathrm{S}_{1}\right)$ and the cardboard printed with a thin layer of UV varnish $\left(\mathrm{S}_{2}\right)$ (values in the brackets represent the coefficient of variation [\%])

\begin{tabular}{c|c|c|c|c|c|c}
\hline \multirow{2}{*}{ Film } & \multicolumn{2}{|c|}{ Breaking effort, $\boldsymbol{\sigma}$ MPa } & \multicolumn{2}{c|}{ Relative elongation, $\boldsymbol{\varepsilon}(\%)$} & \multicolumn{2}{c}{ Absolute elongation, I (cm) } \\
\cline { 2 - 7 } & $\begin{array}{c}\text { Longitudinal } \\
\text { direction }\end{array}$ & $\begin{array}{c}\text { Transverse } \\
\text { direction }\end{array}$ & $\begin{array}{c}\text { Longitudinal } \\
\text { direction }\end{array}$ & $\begin{array}{c}\text { Transverse } \\
\text { direction }\end{array}$ & $\begin{array}{c}\text { Longitudinal } \\
\text { direction }\end{array}$ & $\begin{array}{c}\text { Transverse } \\
\text { direction }\end{array}$ \\
\cline { 2 - 7 } & Min/max & Min/max & Min/max & Min/max & Min/max & Min/max \\
\hline ORACAL (unprinted) & $28.1 / 46$ & $19 / 30.6$ & $170 / 210$ & $130 / 156$ & $27 / 36$ & $19 / 26$ \\
\hline RITRAMA (unprinted) & $42.2 / 68.5$ & $28.5 / 45.9$ & $275 / 310$ & $245 / 268$ & $34 / 42$ & $25 / 32$ \\
\hline ORACAL (printed) & $5.7 / 6.4$ & $7.8 / 9.6$ & $145 / 168$ & $123 / 144$ & $17 / 28$ & $14 / 19$ \\
\hline RITRAMA (printed) & $6.8 / 7.7$ & $9.4 / 11.5$ & $238 / 280$ & $217 / 243$ & $26 / 37$ & $19 / 26$ \\
\hline
\end{tabular}

(min) respectively in the longitudinal and transverse direction for film RITRAMA-145. Some other properties have printed films surfaces. Breaking load $\sigma$ for printed film ORACAL decreases from 6.4 MPa to 5.7 MPa in longitudinal direction, and from 9.6 MPa to 7.8 MPa in transverse direction. For the printed film RITRAMA-145 the difference between the largest and smallest values of $\sigma$ in the longitudinal direction is $0.9 \mathrm{MPa}$ and in the transverse direction is $2.1 \mathrm{MPa}$. It is obviously possible to explain by significant influence of solvent inks on adhesion properties of the films (Havenko \& Sheludko, 2016).

In the manufacturing process and products labeling there are certain deformation of base-substrate and labels polymer coating, which is exposed to external mechanical influences and can cause deformation of the lens. It is known that the degree of deformation is the relative deformation, which is the ratio of the absolute deformation to the original body size:

$\Delta l=l-l_{0}, \varepsilon=\frac{\Delta l}{l_{0}}$

Mechanical stress $\sigma-$ is a physical quantity that is numerically equal to the force of elasticity per unit area of the body section:

$\vec{\sigma}=\frac{d \vec{F}_{n p}}{d S}$

Changes of transverse bodies' sizes characterize by relative transverse compression or tension:

$\varepsilon^{\prime}=\frac{\Delta d}{d_{0}}$

where $d_{0}$ - transverse bodies' sizes before deformation. The ratio of the relative transverse deformation to the longitudinal deformation of the body is called Poisson coefficient:

$\mu=\frac{\varepsilon^{\prime}}{\varepsilon}$

Poisson's coefficient depends on the body material and is one of the constants that characterize body elastic properties. It was considered body that belongs to the curvilinear orthogonal coordinate system $\alpha, \beta, \gamma$. Let unit vectors that are tangent to coordinate lines through accordingly. According to the assumption there is a relationship between coordinates $\alpha, \beta, \gamma$ and Cartesian rectangular coordinates $x, y, z$ (Rehbinder, 1978; Pocius, 2002; Sheludko, 2013):

$x=x(\alpha, \beta, \gamma), y=y(\alpha, \beta, \gamma), z=z(\alpha, \beta, \gamma)$

Curvilinear orthogonal coordinate system is characterized by Lame parameters that are determined by the following formulas:

$$
\begin{aligned}
& \mathrm{H}_{1}^{2}=\left(\frac{\partial x}{\partial \alpha}\right)^{2}+\left(\frac{\partial y}{\partial \alpha}\right)^{2}+\left(\frac{\partial z}{\partial \alpha}\right)^{2}, \mathrm{H}_{2}^{2}=\left(\frac{\partial x}{\partial y}\right)^{2}+\left(\frac{\partial y}{\partial \beta}\right)^{2}+\left(\frac{\partial z}{\partial \beta}\right)^{2} \\
& \mathrm{H}_{3}^{2}=\left(\frac{\partial x}{\partial \gamma}\right)^{2}+\left(\frac{\partial y}{\partial \gamma}\right)^{2}+\left(\frac{\partial z}{\partial \gamma}\right)^{2} .
\end{aligned}
$$

An example of orthogonal coordinates is the Cartesian, cylindrical and other coordinates. As you know, Lame parameters - are independent values and, according to differential geometry, they must satisfy six differential relations (Pysarenko \& Lebedev, 1976; Timoshenko \& Goodier, 1979; Shvab'yuk, 2009):

$\frac{\partial}{\partial \alpha}\left(\frac{1}{\mathrm{H}_{1}} \frac{\partial \mathrm{H}_{2}}{\partial \alpha}\right)+\frac{\partial}{\partial \beta}\left(\frac{1}{\mathrm{H}_{2}} \frac{\partial \mathrm{H}_{1}}{\partial \beta}\right)+\frac{1}{\mathrm{H}_{3}^{2}} \frac{\partial \mathrm{H}_{1}}{\partial \gamma} \frac{\partial \mathrm{H}_{2}}{\partial \gamma}=0$,

$\frac{\partial}{\partial \beta}\left(\frac{1}{\mathrm{H}_{2}} \frac{\partial \mathrm{H}_{3}}{\partial \beta}\right)+\frac{\partial}{\partial \gamma}\left(\frac{1}{\mathrm{H}_{3}} \frac{\partial \mathrm{H}_{2}}{\partial \gamma}\right)+\frac{1}{\mathrm{H}_{3}^{2}} \frac{\partial \mathrm{H}_{2}}{\partial \alpha} \frac{\partial \mathrm{H}_{3}}{\partial \alpha}=0$,

$\frac{\partial}{\partial \gamma}\left(\frac{1}{\mathrm{H}_{3}} \frac{\partial \mathrm{H}_{1}}{\partial \gamma}\right)+\frac{\partial}{\partial \alpha}\left(\frac{1}{\mathrm{H}_{1}} \frac{\partial \mathrm{H}_{3}}{\partial \alpha}\right)+\frac{1}{\mathrm{H}_{2}^{2}} \frac{\partial \mathrm{H}_{3}}{\partial \beta} \frac{\partial \mathrm{H}_{1}}{\partial \beta}=0$,

$\frac{\partial^{2} \mathrm{H}_{1}}{\partial \beta \partial \gamma}-\frac{1}{\mathrm{H}_{2}} \frac{\partial \mathrm{H}_{2}}{\partial \gamma} \frac{\partial \mathrm{H}_{1}}{\partial \beta}-\frac{1}{\mathrm{H}_{3}^{2}} \frac{\partial \mathrm{H}_{3}}{\partial \beta} \frac{\partial \mathrm{H}_{1}}{\partial \gamma}=0$,

$\frac{\partial^{2} \mathrm{H}_{2}}{\partial \gamma \partial \alpha}-\frac{1}{\mathrm{H}_{3}} \frac{\partial \mathrm{H}_{3}}{\partial \alpha} \frac{\partial \mathrm{H}_{2}}{\partial \gamma}-\frac{1}{\mathrm{H}_{1}} \frac{\partial \mathrm{H}_{1}}{\partial \gamma} \frac{\partial \mathrm{H}_{2}}{\partial \alpha}=0$,

$\frac{\partial^{2} \mathrm{H}_{3}}{\partial \alpha \partial \beta}-\frac{1}{\mathrm{H}_{1}} \frac{\partial \mathrm{H}_{1}}{\partial \beta} \frac{\partial \mathrm{H}_{3}}{\partial \alpha}-\frac{1}{\mathrm{H}_{2}} \frac{\partial \mathrm{H}_{2}}{\partial \alpha} \frac{\partial \mathrm{H}_{3}}{\partial \beta}=0$.

Let the body elastically deformed. Then body point with the coordinates $\alpha, \beta, \gamma$ gets moving, which can be represented by three vector projection of full movement in tangent directions to coordinate lines $\alpha, \beta, \gamma$ :

$u_{1}=u_{1}(\alpha, \beta, \gamma), u_{2}=u_{2}(\alpha, \beta, \gamma), u_{3}=u_{3}(\alpha, \beta, \gamma)$

In the theory of nonlinear elasticity it is taken to distinguish between deformation tensors of Grin, Almanzi, 
Hyenki and others. This deformation state of solid bodies we describe with six components of Grin (Sheludko,

2013):

$$
\begin{aligned}
& \varepsilon_{11}=\ell_{11}+\frac{1}{2}\left[\ell_{11}^{2}+\left(\frac{1}{2} \ell_{12}+\omega_{3}\right)^{2}+\left(\frac{1}{2} \ell_{13}-\omega_{2}\right)^{2}\right], \\
& \varepsilon_{22}=\ell_{22}+\frac{1}{2}\left[\ell_{22}^{2}+\left(\frac{1}{2} \ell_{23}+\omega_{1}\right)^{2}+\left(\frac{1}{2} \ell_{21}+\omega_{3}\right)^{2}\right], \\
& \varepsilon_{33}=\ell_{33}+\frac{1}{2}\left[\ell_{33}^{2}+\left(\frac{1}{2} \ell_{31}+\omega_{2}\right)^{2}+\left(\frac{1}{2} \ell_{32}-\omega_{1}\right)^{2}\right], \\
& \varepsilon_{12}=\varepsilon_{21}=\ell_{12}+\ell_{11}\left(\frac{1}{2} \ell_{12}-\omega_{3}\right)+\ell_{22}\left(\frac{1}{2} \ell_{12}+\omega_{3}\right)+\left(\frac{1}{2} \ell_{13}-\omega_{2}\right)\left(\frac{1}{2} \ell_{23}+\omega_{1}\right), \\
& \varepsilon_{13}=\varepsilon_{31}=\ell_{13}+\ell_{33}\left(\frac{1}{2} \ell_{13}-\omega_{2}\right)+\ell_{11}\left(\frac{1}{2} \ell_{13}+\omega_{2}\right)+\left(\frac{1}{2} \ell_{23}-\omega_{1}\right)\left(\frac{1}{2} \ell_{12}+\omega_{3}\right), \\
& \varepsilon_{23}=\varepsilon_{32}=\ell_{23}+\ell_{22}\left(\frac{1}{2} \ell_{23}-\omega_{1}\right)+\ell_{33}\left(\frac{1}{2} \ell_{23}+\omega_{1}\right)+\left(\frac{1}{2} \ell_{21}-\omega_{3}\right)\left(\frac{1}{2} \ell_{31}+\omega_{2}\right),
\end{aligned}
$$

where

$\ell_{11}=\frac{1}{H_{1}} \frac{\partial u_{1}}{\partial \alpha}+\frac{1}{H_{1} H_{2}} \frac{\partial H_{1}}{\partial \beta} u_{2}+\frac{1}{H_{1} H_{3}} \frac{\partial H_{1}}{\partial \gamma} u_{3}$,

$\ell_{22}=\frac{1}{H_{2}} \frac{\partial u_{2}}{\partial \beta}+\frac{1}{H_{2} H_{3}} \frac{\partial H_{2}}{\partial \gamma} u_{3}+\frac{1}{H_{2} H_{1}} \frac{\partial H_{2}}{\partial \alpha} u_{1}$,

$\ell_{33}=\frac{1}{H_{3}} \frac{\partial u_{3}}{\partial \gamma}+\frac{1}{H_{1} H_{3}} \frac{\partial H_{3}}{\partial \alpha} u_{1}+\frac{1}{H_{3} H_{2}} \frac{\partial H_{3}}{\partial \beta} u_{2}$,

$\ell_{12}=\ell_{21}=\frac{H_{2}}{H_{1}} \frac{\partial}{\partial \alpha}\left(\frac{u_{2}}{H_{2}}\right)+\frac{H_{1}}{H_{2}} \frac{\partial}{\partial \beta}\left(\frac{u_{1}}{H_{1}}\right)$,

$\ell_{13}=\ell_{31}=\frac{H_{3}}{H_{1}} \frac{\partial}{\partial \alpha}\left(\frac{u_{3}}{H_{3}}\right)+\frac{H_{1}}{H_{3}} \frac{\partial}{\partial \gamma}\left(\frac{u_{2}}{H_{1}}\right)$,

$\ell_{23}=\ell_{32}=\frac{H_{2}}{H_{3}} \frac{\partial}{\partial \gamma} \cdot\left(\frac{u_{2}}{H_{2}}\right)+\frac{H_{3}}{H_{2}} \frac{\partial}{\partial \beta}\left(\frac{u_{3}}{H_{3}}\right)$

The rotation angles of volume element around the coordinate axes look like:

$$
\begin{aligned}
& \omega_{1}=\frac{1}{2 H_{2} H_{3}}\left[\frac{\partial}{\partial \beta}\left(H_{3} u_{2}\right)-\frac{\partial}{\partial \gamma}\left(H_{2} u_{2}\right)\right], \\
& \omega_{2}=\frac{1}{2 H_{1} H_{3}}\left[\frac{\partial}{\partial \gamma}\left(H_{1} u_{1}\right)-\frac{\partial}{\partial \alpha}\left(H_{3} u_{3}\right)\right], \\
& \omega_{3}=\frac{1}{2 H_{1} H_{2}}\left[\frac{\partial}{\partial \alpha}\left(H_{2} u_{2}\right)-\frac{\partial}{\partial \beta}\left(H_{1} u_{1}\right)\right] .
\end{aligned}
$$

Relative elongation in directions $\alpha, \beta, \gamma$ is calculated by the formula:

$$
E_{1}=\sqrt{1+2 \varepsilon_{11}}-1, E_{2}=\sqrt{1+2 \varepsilon_{22}}-1, E_{3}=\sqrt{1+2 \varepsilon_{23}}-1 .
$$

Shift angles between layers of labels:

$$
\mathrm{n} \varphi_{12}=\frac{\varepsilon_{12}}{\left(1+E_{1}\right)\left(1+E_{2}\right)}, \sin \varphi_{23}=\frac{\varepsilon_{23}}{\left(1+E_{2}\right)\left(1+E_{3}\right)}, \sin \varphi_{13}=\frac{\varepsilon_{13}}{\left(1+E_{1}\right)\left(1+E_{3}\right)}
$$

Unit vector $\vec{K}_{1}^{*}, \vec{K}_{2}^{*}, \vec{K}_{3}^{*}$, tangent to the lines $\alpha, \beta, \gamma$ in the body after the deformation found through $\vec{K}_{1}, \vec{K}_{2}, \vec{K}_{3}$ by the formulas:

$$
\begin{aligned}
& \vec{K}_{1}^{*}=\frac{1}{1+E_{1}}\left[\left(1+\ell_{11}\right) \vec{K}_{1}+\left(\frac{1}{2} \ell_{12}+\omega_{3}\right) \vec{K}_{2}+\left(\frac{1}{2} \ell_{13}-\omega_{2}\right) \vec{K}_{3}\right], \\
& \vec{K}_{2}^{*}=\frac{1}{1+E_{2}}\left[\left(\frac{1}{2} \ell_{12}-\omega_{3}\right) \vec{K}_{1}+\left(1+\ell_{22}\right) \vec{K}_{2}+\left(\frac{1}{2} \ell_{23}+\omega_{1}\right) \vec{K}_{3}\right], \\
& \vec{K}_{3}^{*}=\frac{1}{1+E_{3}}\left[\left(\frac{1}{2} \ell_{13}+\omega_{2}\right) \vec{K}_{1}+\left(\frac{1}{2} \ell_{23}-\omega_{1}\right) \vec{K}_{2}+\left(1+\ell_{33}\right) \vec{K}_{3}\right] .
\end{aligned}
$$

It should be also written equation of deformation com- patibility. Components $R_{\text {mnpq }}$ of Riemann-Kristofel tensor should be zero. Since there are six independent components of Riemann-Kristofel tensor, there are fulfilled conditions:

$R_{1212}=0, R_{1313}=0, R_{2323}=0$,
$R_{1213}=0, R_{2123}=0, R_{3132}=0$.

Equations (15), which must satisfy the deformation components $\varepsilon_{i j}$, are necessary and sufficient conditions that the configuration of nondeformed and deformed body's state belongs to Euclidean space. With equation of compatibility implies that the movement $u_{\cdot 1}, u_{2}, u_{3}$ must have continuous derivatives to third order inclusive. Stress state in theory of finite deformations (Rehbinder, 1978; Pocius, 2002; Sheludko, 2013) can be characterized by different stress tensor: symmetric and asymmetric, belonging to one area in the not deformed and deformed states. For information about these tensors can be found in (Pocius, 2002). It was consider stress tensor, which components belong to the nondeformed state. If it will be consider the elementary volume element in the system of coordinates $\alpha, \beta, \gamma$ then on it facets will be such tension: on facet on facet $\alpha=$ const : $\sigma_{11}, \sigma_{21}, \sigma_{31}$, on facet $\beta=$ const $: \sigma_{22}, \sigma_{12}, \sigma_{32}$, on facet $\gamma=$ const $: \sigma_{33}, \sigma_{13}, \sigma_{23}$.

These stresses are called true stress. Related stresses have the property of reciprocity, which are true by relationships:

$\sigma_{i j}=\sigma_{j i},_{(i j=1,2,3)}$

Let the elastic regard body under the applied force is in equilibrium. Equilibrium conditions of elementary deformed volume body in curvilinear orthogonal coordinate system $\alpha, \beta, \gamma$ to which the body lies in the not deformed state have the form (Rehbinder, 1978; Pocius, 2002; Sheludko, 2013):

$\frac{\partial}{\partial \alpha}\left(H_{2} H_{3} S_{11}\right)+\frac{\partial}{\partial \beta}\left(H_{1} H_{3} S_{21}\right)+\frac{\partial}{\partial \gamma}\left(H_{1} H_{2} S_{31}\right)-H_{3} \frac{\partial H_{2}}{\partial \alpha} S_{22}-$

$-H_{2} \frac{\partial H_{3}}{\partial \alpha} S_{33}+H_{3} \frac{\partial H_{1}}{\partial \beta} S_{12}+H_{2} \frac{\partial H_{1}}{\partial \gamma} S_{13}+H_{1} H_{2} H_{3} P_{1}^{*}=0 ;$

$\frac{\partial}{\partial \alpha}\left(H_{2} H_{3} S_{12}\right)+\frac{\partial}{\partial \beta} \cdot\left(H_{3} H_{1} S_{22}\right)+\frac{\partial}{\partial \gamma}\left(H_{1} H_{2} S_{23}\right)+H_{1} \frac{\partial H_{2}}{\partial \gamma} S_{23}+$

$+H_{3} \frac{\partial H_{2}}{\partial \alpha} S_{21}-H_{1} \frac{\partial H_{3}}{\partial \beta} S_{33}-H_{3} \frac{\partial H_{1}}{\partial \beta} S_{11}+H_{1} H_{2} H_{3} P_{2}^{*}=0 ;$

$\frac{\partial}{\partial \alpha}\left(H_{2} H_{3} S_{12}\right)+\frac{\partial}{\partial \beta}\left(H_{3} H_{1} S_{23}\right)+\frac{\partial}{\partial \gamma}\left(H_{1} H_{2} S_{33}\right)+H_{2} \frac{\partial H_{3}}{\partial \alpha} S_{31}+$ $+H_{1} \frac{\partial H_{3}}{\partial \beta} S_{32}-H \frac{\partial H_{1}}{\partial \gamma} S_{11}-H_{1} \frac{\partial H_{2}}{\partial \gamma} S_{22}+H_{1} H_{2} H_{3} P_{3}^{*}=0$,

where $P_{i}^{*}$ - projection of mass forces on $\vec{K}_{1}, \vec{K}_{2}, \vec{K}_{3}$. Volume force that acting on regard rectangular parallelepiped:

$\vec{F} d V^{*}=\vec{P} D d V=\vec{P}^{*} d V$, 
where $d V^{*}$ - volume of oblique parallelepiped;

$d V=H_{1} H_{2} H_{3} d \alpha d \beta d \gamma$

where $d V$ - the volume of a rectangular parallelepiped.

According to (Rehbinder, 1978; Pocius, 2002; Sheludko, 2013):

$P_{i}^{*}=P_{i} D$,

Components $S_{i j}$ in equations of equilibrium form the asymmetric tensor:

$$
\begin{aligned}
& S_{11}=\sigma_{11}^{*}\left(1+\ell_{12}\right)+\sigma_{12}^{*}\left(\frac{1}{2} \ell_{12}-\omega_{3}\right)+\sigma_{13}^{*}\left(\frac{1}{2} \ell_{13}+\omega_{2}\right), \\
& S_{12}=\sigma_{11}^{*}\left(\frac{1}{2} \ell_{12}+\omega_{3}\right)+\sigma_{12}^{*}\left(1+\ell_{22}\right)+\sigma_{13}^{*}\left(\frac{1}{2} \ell_{23}-\omega_{1}\right), \\
& S_{13}=\sigma_{11}^{*}\left(\frac{1}{2} \ell_{13}-\omega_{2}\right)+\sigma_{12}^{*}\left(\frac{1}{2} \ell_{23}+\omega_{1}\right)+\sigma_{13}^{*}\left(1+\ell_{33}\right), \\
& S_{21}=\sigma_{21}^{*}\left(1+\ell_{11}\right)+\sigma_{22}^{*}\left(\frac{1}{2} \ell_{1}-\omega_{3}\right)+\sigma_{23}^{*}\left(\frac{1}{2} \ell_{13}+\omega_{2}\right), \\
& S_{22}=\sigma_{21}^{*}\left(\frac{1}{2} \ell_{12}+\omega_{3}\right)+\sigma_{22}^{*}\left(1+\ell_{22}\right)+\sigma_{23}^{*}\left(\frac{1}{2} \ell_{23}-\omega_{1}\right), \\
& S_{23}=\sigma_{21}^{*}\left(\frac{1}{2} \ell_{12}+\omega_{2}\right)+\sigma_{22}^{*}\left(\frac{1}{2} \ell_{23}-\omega_{1}\right)+\sigma_{23}^{*}\left(1+\ell_{33}\right), \\
& S_{31}=\sigma_{31}^{*}\left(1+\ell_{12}\right)+\sigma_{32}^{*}\left(\frac{1}{2} \ell_{12}-\omega_{3}\right)+\sigma_{33}^{*}\left(\frac{1}{2} \ell_{13}+\omega_{2}\right), \\
& S_{32}=\sigma_{31}^{*}\left(\frac{1}{2} \ell_{12}+\omega_{3}\right)+\sigma_{32}^{*}\left(1+\ell_{22}\right)+\sigma_{33}^{*}\left(\frac{1}{2} \ell_{23}-\omega_{1}\right), \\
& S_{33}=\sigma_{31}^{*}\left(\frac{1}{2} \ell_{13}-\omega_{2}\right)+\sigma_{32}^{*}\left(\frac{1}{2} \ell_{23}+\omega_{1}\right)+\sigma_{33}^{*}\left(1+\ell_{33}\right),
\end{aligned}
$$

The values $\sigma_{i j}^{*}$ are called generalized stresses. They are connected with the true stress as follows:

$\sigma_{i j}^{*}=\frac{\sigma_{i j}}{1+E_{j}} \frac{S_{i}^{*}}{S_{i}}$,

Here $S_{i}$ area of rectangular elementary zone beforedeformation; $S_{i}^{*}$ area of zone (curved quadrangle) after deformation. According to (Rehbinder, 1978; Pocius, 2002; Sheludko, 2013; ) their ratio is determined by formulas:

$$
\begin{aligned}
& \frac{S_{1}^{*}}{S_{1}}=\sqrt{\left(1+2 \varepsilon_{22}\right)\left(1+2 \varepsilon_{33}\right)-\varepsilon_{23}^{2}}, \frac{S_{2}^{*}}{S_{2}}=\sqrt{\left(1+2 \varepsilon_{33}\right)\left(1+2 \varepsilon_{11}\right)-\varepsilon_{31}^{2}}, \\
& \frac{S_{3}^{*}}{S_{3}}=\sqrt{\left(1+2 \varepsilon_{11}\right)\left(1+2 \varepsilon_{22}\right)-\varepsilon_{12}^{2}} .
\end{aligned}
$$

It was considered the connection between stresses and deformations. Writing the physical correlation that characterizes properties of the elastic body we will come from general thermodynamic laws. According to the first law of thermodynamics:

$\delta E=\delta^{\prime} A+\delta^{\prime} Q$

where $\delta E$ - increase of internal energy; $\delta^{\prime} Q-$ elementary quantity of heat; $\delta^{\prime} A$ - elementary work of external forces. All values in ratios (26) belonging to one of the initial body volume. If the inverse process

$\delta^{\prime} Q=T \delta S$

where $T$ - absolute body temperature; $\delta S$ - increase of entropy,

$\delta^{\prime} A=\sigma_{i j}^{*} \delta \varepsilon_{i j}$

Therefore, to increase internal energy we have:

$\delta E=T \delta S+\sigma_{i j}^{*} \delta \varepsilon_{i j}$.

Using free energy $F=E-T \delta$, we get:

$\delta F=S \delta T-\sigma_{i j}^{*} \delta E_{i j}$.

Assuming that the internal energy $E=E\left(\varepsilon_{i j}, S\right)$ of the body is a function of deformations tensor components and entropy $S$, and free energy $F=F\left(\varepsilon_{i j}, T\right)$ - is a function of deformations tensor components and temperature, from (29) and (30) we obtain:

$\sigma_{i j}^{*}=\left(\frac{\partial E}{\partial \varepsilon_{i j}}\right)_{S=c o n s t}, T=\left(\frac{\partial E}{\partial S}\right)_{\varepsilon_{i j}=c o n s t} ;$

$\sigma_{i j}^{*}=\left(\frac{\partial F}{\partial \varepsilon_{i j}}\right)_{T=\text { const }}, S=-\left(\frac{\partial F}{\partial T}\right)_{\varepsilon_{i j}=\text { const }}$.

During adiabatic $\left(\delta^{\prime} Q=0\right)$ or isothermal ( $T=$ const $)$ process it can be introduce a function $\phi$, called the specific potential energy of the body that depends on deformations tensor components. In this regard:

$\sigma_{i j}^{*}=\frac{1}{2}\left(\frac{\partial}{\partial \varepsilon_{i j}}+\frac{\partial}{\partial \varepsilon_{j i}}\right) \phi\left(\varepsilon_{11}, \varepsilon_{12}, \varepsilon_{21}, \ldots, \varepsilon_{33}\right)$.

Choosing of expressions for internal and free energy, and for the elastic potential is difficult task. Assuming that the natural state of the body hasn't stress and deformation for values $E\left(\varepsilon_{i j}, S\right)$ and $F\left(\varepsilon_{i j}, T\right)$ the general case of nonlinear elastic anisotropic body can be represented as a power series:

$$
\begin{aligned}
& E=E(0, S)+\frac{1}{2} C_{i j k e}^{S} \varepsilon_{i j} \varepsilon_{k e}+\frac{1}{6} C_{i j k e m n}^{S} \varepsilon_{i j} \varepsilon_{k e} \varepsilon_{m n}+\ldots \\
& F=F(0, T)+\frac{1}{2} C_{i j k e}^{T} \varepsilon_{i j} \varepsilon_{k e}+\frac{1}{6} C_{i j k e m n}^{T} \varepsilon_{i j} \varepsilon_{k e} \varepsilon_{m n}+\ldots .
\end{aligned}
$$

Coefficients included in these schedules, are functions(35) of entropy or temperature and are called according isothermal and adiabatic constant of second, third, etc. orders. They are determined by formulas:

$$
C_{i j k e}^{S}=\left(\frac{\partial^{n} E\left(\varepsilon_{i j}, S\right)}{\partial \varepsilon_{i j} \partial \varepsilon_{k e} \cdots}\right)_{S}
$$


$C_{i j k e}^{T}=\left(\frac{\partial^{n} F\left(\varepsilon_{i j}, T\right)}{\partial \varepsilon_{i j} \partial \varepsilon_{k e} \cdots}\right)_{T}$

For elastic potential (in the general case, non-linear anisotropic elastic body) it is fair expression:

$\phi\left(\varepsilon_{i j}\right)=\frac{1}{2} K_{i j k e} \varepsilon_{i j} \varepsilon_{k e}+\frac{1}{6} K_{i j k e m n} \varepsilon_{i j} \varepsilon_{k e} \varepsilon_{m n}+\ldots$,

where $K_{i j k e}=\frac{\partial^{n} \phi\left(\varepsilon_{i j}\right)}{\partial \varepsilon_{i j} \partial \varepsilon_{k e}}$,

coefficient of elasticity of the second, third, etc. orders.

In the case of an isotropic body internal and free energy is a function of temperature and deformation tensor invariants, and elastic capacity - only deformations tensor invariants, that is:

$E=E\left(S, A_{1}, A_{2}, A_{3}\right), F=F\left(T, A_{1}, A_{2}, A_{3}\right), \phi=\phi\left(A_{1}, A_{2}, A_{3}\right)$,

where $A_{i}(i=1,2,3)$ - algebraic invariants of the Grin deformations tensor:

$A_{1}=\varepsilon_{i i}, A_{2}=\varepsilon_{i j} \varepsilon_{j i}, A_{3}=\varepsilon_{i j} \varepsilon_{j k} \varepsilon_{k i}$.

In formulas (39), as known (Rehbinder, 1978; Sheludko, 2013), for the basic invariants can be taken another invariants system. Elasticity coefficients of second order are describing the linear relationship between stresses and deformations. Constants of third order are characterizing the nonlinearity quadratic of the environment, the fourth - cubic etc.

It was considered boundary conditions on the movement and tension. Boundary conditions in movements on the part of body surface $S_{l}$. Boundary conditions on the surface $S_{2}$ tensions will be written in the form (Rehbinder, 1978; Pocius, 2002; Sheludko, 2013).

$S_{11} \cos \left(\bar{n}, \bar{K}_{1}\right)+S_{21} \cos \left(\bar{n}, \bar{K}_{2}\right)+S_{31} \cos \left(\bar{n}, \bar{K}_{3}\right)=f_{1}^{*}$,

$S_{12} \cos \left(\bar{n}, \bar{K}_{1}\right)+S_{22} \cos \left(\bar{n}, \bar{K}_{2}\right)+S_{32} \cos \left(\bar{n}, \bar{K}_{3}\right)=f_{2}^{*}$,

$S_{13} \cos \left(\bar{n}, \bar{K}_{1}\right)+S_{23} \cos \left(\bar{n}, \bar{K}_{2}\right)+S_{33} \cos \left(\bar{n}, \bar{K}_{3}\right)=f_{3}^{*}$,

where components $S_{i j}$ - determined by formulas (23) and components of surface forces $f_{j}$ are as follows:

$f_{i}^{*}=\frac{S_{n}^{*}}{S_{n}} f_{j}$.

Here $f_{i}$ components of surface forces in directions $\bar{K}_{1}, \bar{K}_{2}, \bar{K}_{3}$, and

$\left(\frac{S_{n}^{*}}{S_{n}}\right)=D^{2}\left[\begin{array}{l}q^{11} \cos ^{2}\left(\bar{n}, \bar{K}_{1}\right)+q^{22} \cos ^{2}\left(\bar{n}, \bar{K}_{2}\right)+q^{33} \cos ^{2}\left(\bar{n}, \bar{K}_{3}\right)+ \\ +2 q^{13} \cos \left(\bar{n}, \bar{K}_{1}\right) \cos \left(\bar{n}, \bar{K}_{3}\right)+2 q^{2} \cos \left(\bar{n}, \bar{K}_{1}\right) \cos \left(\bar{n}, \bar{K}_{2}\right)+ \\ +2 q^{23} \cos \left(\bar{n}, \bar{K}_{2}\right) \cos \left(\bar{n}, \bar{K}_{3}\right)\end{array}\right]$, where

$$
\begin{aligned}
& q^{11}=\frac{1}{D^{2}}\left[\left(1+2 \varepsilon_{22}\right)\left(1+2 \varepsilon_{33}\right)-\varepsilon_{23}^{2}\right] ; \\
& q^{22}=\frac{1}{D^{2}}\left[\left(1+2 \varepsilon_{11}\right)\left(1+2 \varepsilon_{33}\right)-\varepsilon_{13}^{2}\right] ; \\
& q^{33}=\frac{1}{D^{2}}\left[\left(1+2 \varepsilon_{11}\right)\left(1+2 \varepsilon_{22}\right)-\varepsilon_{12}^{2}\right] ; \\
& q^{12}=-\frac{1}{D^{2}}\left[\varepsilon_{12}\left(1+2 \varepsilon_{33}\right)-\varepsilon_{23} \varepsilon_{13}\right] ; \\
& q^{13}=-\frac{1}{D^{2}}\left[\varepsilon_{13}\left(1+2 \varepsilon_{22}\right)-\varepsilon_{12} \varepsilon_{23}\right] ; \\
& q^{23}=-\frac{1}{D^{2}}\left[\varepsilon_{23}\left(1+2 \varepsilon_{11}\right)-\varepsilon_{21} \varepsilon_{13}\right] ;
\end{aligned}
$$

In ratios $\left(\bar{n}, \bar{K}_{1}\right),\left(\bar{n}, \bar{K}_{2}\right),\left(\bar{n}, \bar{K}_{3}\right)$ - angles, those are formed by the normal to the platform, which we consider (in its position to the deformation) and the unit vector $\bar{K}_{1}, \bar{K}_{2}, \bar{K}_{3}$. On the surface of the body it can be set mixed boundary conditions (Olijnyk \& Ogirko, 2016). For example, in Cartesian rectangular coordinate system with boundary conditions of the first order we have:

$L_{m}(N, F) \equiv \frac{\partial}{\partial x_{i}}\left[\sigma_{i j}\left(\delta_{k j}+\frac{\partial u_{k}}{\partial x_{j}}\right)\right]=0_{m=1,2,3} ;$

$L_{4}(N, F) \equiv \frac{\partial}{\partial x_{i}}\left(\lambda_{t} q^{i j} \frac{\partial T}{\partial x_{j}}\right)=0$;

$H_{m}(N, F) \equiv u_{m}-u_{m}^{S}=0$

$H_{T}(N, F) \equiv T_{S}-T_{S}^{S}=0$,

where $u_{m}^{S}, T_{S}^{S}$ - set move and the temperature on the surface of the body.

Thus, the horizontal level of resin that applied to the image's surface is controlled in the labels manufacturing process. Resin fills this surface and creates a unique transparent dome as a result of display of plastic and highly elastic deformation. On these deformations affect the chemical nature of polyurethane and epoxy resins, their physical and mechanical properties.

\section{Conclusions}

As a result of modeling and experimental researches it was confirmed that for the investigated volume labels produced on self-adhesive PVC film type ORACAL and RITRAMA-145 exists linear dependence of deformation and stress, which they are exposed in the process of labeling products. It was found that RITRAMA-145 more resistant to destruction by 1.5 times than ORACAL. Deformation properties of base-substrate differ in transverse and longitudinal directions, which should be considered when making labels. On the deformation properties of self-adhesive material impact method of applying on them printed image. The value of deformations tensor depends on the physical and chemical nature of the resin, the thickness of the lens of volume label. 


\section{References}

GOST (1981) 14236-81. Polymer films: Tensile strength test method. Moscow, National Russian Standards.

Havenko, S. \& Sheludko, S. (2016) Research the process of forming volume polymer coating based on epoxy resin on the surface of labels-stickers. Book Qualilogy. 1, 17-28.

Olijnyk, R. \& Ogirko, O. (2016) Information technology of information systems data processing with variable structure and options. Computer Technologies of Printing. 1 (35), 87-97.

Pocius, A. V. (2002) Adhesion and Adhesives Technology: An Introduction. 2nd ed. Munich, Hanser.

Pysarenko, G. S. \& Lebedev, A. A. (1976) Deformation and strength of materials under complex stress state. Kiev, Scientific Thought Publications.

Rehbinder, P. A. (1978) Selected Works: Surface Phenomena in Dispersed Systems. Colloid Chemistry. Moscow, Nauka.

Sheludko, S. (2013) Influence of the polymer coating thickness of the volume label on its quality characteristics of color images. Book Qualilogy. 1, 37-44.

Shvab'yuk, V. (2009) Strength of materials: Manual guidance for students. Kyiv.

Timoshenko, S. P. \& Goodier, J. N. (1979) Elasticity Theory. Moscow, Nauka.

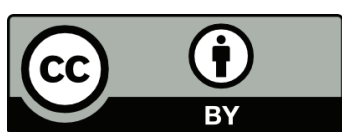

(C) 2017 Authors. Published by the University of Novi Sad, Faculty of Technical Sciences, Department of Graphic Engineering and Design. This article is an open access article distributed under the terms and conditions of the Creative Commons Attribution license 3.0 Serbia (http://creativecommons.org/licenses/by/3.0/rs/). 